\title{
Analyst
}

A) Check for updates

Cite this: Analyst, 2021, 146, 4535

\section{A portable droplet microfluidic device for cortisol measurements using a competitive heterogeneous assay $\uparrow$}

\author{
Gareth W. H. Evans, ${ }^{\text {a, b } W a h i d a ~ T . ~ B h u i y a n, ~(D) ~ a ~ S u s a n ~ P a n g, ~}{ }^{c}$ Brett Warren, ${ }^{d}$ \\ Kyriacos Makris, ${ }^{d}$ Sharon Coleman, ${ }^{d}$ Sammer-ul Hassan (iD) and Xize Niu (D)*a,b,d
}

\begin{abstract}
Point-of-care monitoring of chemical biomarkers in real-time holds great potential in rapid disease diagnostics and precision medicine. However, monitoring is still rare in practice, as the measurement of biomarkers often requires time consuming and labour intensive assay procedures such as enzyme linked immunosorbent assay (ELISA), which pose a challenge to an autonomous point-of-care device. This paper describes a prototype device capable of performing ELISA autonomously and repeatedly in a high frequency using droplet microfluidics. Driven by a specially designed peristaltic pump, the device can collect liquid samples from a reservoir, produce trains of droplets, complete magnetic bead based ELISA protocols and provide readouts with colourimetric measurement. Here, cortisol was chosen as a target analyte as its concentration in the human body varies on a circadian rhythm which may be perturbed by disease. The prototype device draws in and analyses $350 \mathrm{~nL}$ of the sample containing free bioactive cortisol every 10 seconds, with a sample-to-signal time of 10 minutes, and measures favourably in the analytical range of 3.175-100 $\mathrm{ng} \mathrm{ml}^{-1}$, with reliably lower variability compared with the well plate based assay. As most ELISA type assays share similar procedures, we envisage that this approach could form a platform technology for measurement or even continuous monitoring of biomarkers in biological fluids at the point-of-care.
\end{abstract}

Received 18th April 2021 Accepted 24th May 2021

DOI: 10.1039/d1an00671a

rsc.li/analyst
Microfluidics allows for the precision handling of liquid samples and miniaturisation of laboratory assays and detection technologies. While microfluidics is still largely seen as a laboratory tool to be supported by bulky equipment such as syringe pumps, valves and microscopes, recent advances in 3D printing ${ }^{9,10}$ and miniaturisation ${ }^{11,12}$ have led to more portable platforms ${ }^{13}$ and wearable microfluidic systems ${ }^{14}$ for sensing and monitoring.

Compared to implanted electrochemical sensors, microfluidic sensors can measure biomarkers ex vivo with a low risk of foreign body response and signal drift. ${ }^{15,16}$ Importantly, for many biomarkers and therapeutic drugs that we are interested in, biochemistry or molecular biology assays already exist either commercially or in research and clinical laboratories. These assays could be readily miniaturised and thus dramatically reduce the time and cost required for developing new microfluidic sensors.

Recently, Nightingale et al. have demonstrated a wearable microfluidic sensor employing a droplet flow regime integrating mix-and-read assays for glucose and lactate monitoring. ${ }^{17}$ Here we further expand the capabilities of this droplet microfluidics sensor to the measurement of protein molecules using more complex assays such as enzyme-linked immunosorbent assay (ELISA), one of the workhorses in biomedical studies. 
The potential of the magnetic bead based ELISA in droplet microfluidics has been studied earlier, either by moving magnetic beads between 'static droplets' trapped in 'slip chips', ${ }^{18}$ or by moving beads between droplets pre-generated by a syringe pump and 3-axis robot. ${ }^{19-21}$ This enables the implementation of heterogeneous assays including sample purification and multiple washing and assay steps. However, previously reported systems rely on large instrumentation and complex operation of pumps and valves ${ }^{20,21}$ making them less suitable for PoC applications. Here we present an autonomous and portable sensor device prototype for continuous ELISA assays, and apply the device for high frequency cortisol measurement as an example application.

Cortisol, as a type of glucocorticoid, can increase glucose production for energy and metabolic needs, thereby playing an important role in the homeostasis of cardiovascular, immune, renal, skeletal and endocrine systems. ${ }^{22-25}$ In the body, cortisol exists in two formats: free bioactive cortisol, which makes up $3-5 \%$ of the total with the remainder bound to the plasma protein corticosteroid binding globulin (CBG or transcortin) or albumin. Both forms of cortisol (bound and unbound) are present in blood serum, while saliva and urine only contain the unbound form along with its metabolites. ${ }^{24}$

Like many hormones in the body, cortisol secretion varies on a 24-hour circadian rhythm, mediated by changes in metabolic activity. In humans, total serum cortisol can range up to $600 \mathrm{ng} \mathrm{mL}{ }^{-1}$ with standard levels of $140.60 \pm 9.62 \mathrm{ng} \mathrm{mL} \mathrm{m}^{-1}$ in males and $101.03 \pm 8.61 \mathrm{ng} \mathrm{mL^{-1 }}$ in females. ${ }^{26}$ Under normal conditions, cortisol levels peak in the early morning (30 minutes after waking), are at half of the peak levels by late afternoon, and become negligible by midnight. ${ }^{27}$

Other than the day-night cycle, controllable factors such as eating patterns and physical activity can also affect cortisol levels in the body. Psychological/emotional stress is recognised as one of the biggest factors triggering systemic cortisol variation and hence cortisol is popularly called a "stresshormone". ${ }^{28}$ Ongoing stress may cause continuous stimulation of the hypothalamic pituitary adrenal (HPA) axis function, by overriding the inhibitory action of cortisol and may even eliminate the cyclic nature of cortisol causing it to remain elevated throughout the day. ${ }^{29}$ Although moderate homeostatic alterations in the HPA axis are beneficial for physiological and psychological development, ${ }^{30}$ a prolonged abnormal increase of cortisol could inhibit inflammation, depress the immune system, increase fatty and amino acid levels in blood, and even lead to chronic fatigue syndrome, fibromyalgia and many autoimmune disorders. Excessive cortisol levels contribute to the development of Cushing's disease (symptoms: obesity, fatigue and bone fragility) ${ }^{31}$ while continuous low levels of cortisol lead to Addison's disease (symptoms: weight loss, fatigue, and darkening of skinfolds and scars). ${ }^{32}$ In sports science, cortisol is also an important biomarker indicating body condition and training effects in elite athletes. ${ }^{33}$

Currently cortisol is commonly measured using immunoassay protocols in single time snapshots. ${ }^{22}$ However, this does not provide the full picture due to the circadian rhythm or a true representation of cortisol levels in a normal or stressed state. When more intensive continuous monitoring is required for diagnosis or monitoring of treatments, the current procedures are particularly cumbersome requiring patient admission for the time of the study and labour-intensive sample (blood/saliva/urine) collection and offline analysis, ${ }^{34-36}$ which is also prone to introduce additional errors in the pre-analytical stage, ${ }^{37-40}$ especially when patients collect their own samples.

The portable sensor prototype presented here will miniaturise and automate the whole cortisol assay process from sample collection to the magnetic bead based ELISA and measurement, thereby providing a high frequency readout of cortisol levels in biological samples. The sensor is built around a 'flowing droplet' approach, integrating a novel droplet train generator, a magnetic trap, an optical detector and a control system into one single device. This forms a platform technology that can also be used for the measurement and monitoring of other biomarkers requiring multiple step assays.

\section{Assay and methods}

\section{Functionalizing magnetic beads with antibodies}

Magnetic microparticles (Tosylactivated Dynabeads MyOne, purchased from Invitrogen, UK) were coated with anti-cortisol3-BSA antibody (Calbioreagents, CA, US). Briefly, $250 \mu \mathrm{L}$ of original magnetic bead suspension $\left(100 \mathrm{mg} \mathrm{mL}^{-1}\right)$ were rinsed twice with $1 \mathrm{~mL}$ of coating buffer $(0.1 \mathrm{M}$ sodium borate buffer, $\mathrm{pH}$ 9.5). The beads were re-suspended in $100 \mu \mathrm{L}$ coating buffer and mixed thoroughly using a vortex mixer. $500 \mu \mathrm{g}$ of coating antibody were reconstituted in $12.1 \mu \mathrm{L}$ of phosphate buffered saline (PBS), and then mixed with $722.9 \mu \mathrm{L}$ of coating buffer, $100 \mu \mathrm{L}$ of prepared bead suspension and $415 \mu \mathrm{L}$ of $3 \mathrm{M}$ ammonium sulphate solution. The mixture was incubated at room temperature with a slow tilt rotation for 24 hours. The beads were then removed from the coating buffer and suspended in $1250 \mu \mathrm{l}$ of blocking buffer (PBS with $2 \% \mathrm{w} / \mathrm{v}$ bovine serum albumin and $0.05 \%$ Tween) and incubated for another 24 hours with a slow tilt rotation. Finally, the beads were washed twice and re-suspended in storage buffer (PBS, 0.1\% bovine serum albumin and $0.05 \%$ Tween), giving a final concentration of $1 \mathrm{mg} \mathrm{mL}^{-1}$ assuming a $100 \%$ recovery. The bead suspension can be used directly or stored in a fridge at $5{ }^{\circ} \mathrm{C}$ for preservation. All reagents, unless otherwise stated, were purchased from Sigma Aldrich. A set of neodymium magnets were used to retain the beads when washing and re-suspending them.

\section{Competitive heterogeneous cortisol assay in the well plate}

Before integrating into the droplet microfluidics platform, the cortisol assay was first developed for a standard well plate procedure as illustrated in Fig. 1a to generate a calibration curve. $0.1 \mathrm{mg}$ of the antibody-conjugated beads were dispensed into individual wells within a 96-well skirted PCR plate and placed onto a magnetic plate stand for 2 minutes. The resultant 
a)

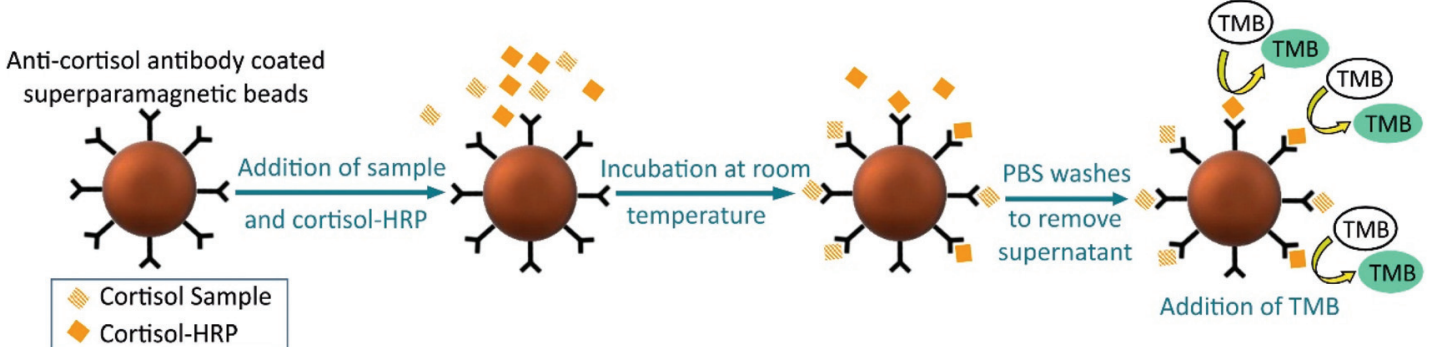

b)

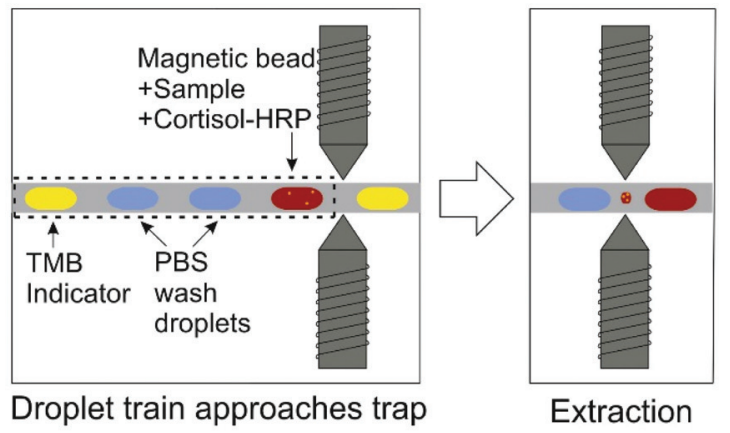

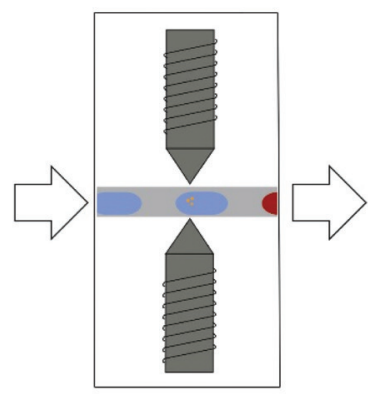

Passing through wash

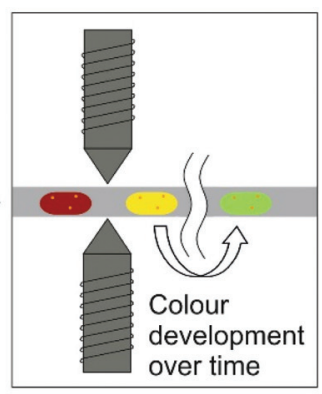

Redispersion in coloured indicator

Fig. 1 Schematic of competitive heterogeneous cortisol assay in both the well plate (a) and droplet flow (b). From left to right, (a) depicts the process of adding sample cortisol and cortisol-HRP mixture to the antibody functionalised bead suspension. Sample cortisol competes with cortisol-HRP to form the final assay complex. After washing in PBS to remove any unbound cortisol and cortisol-HRP, tetramethylbenzidine (TMB) was further added and catalysed by HRP from its native diamine form into a blue-green, one electron oxidation state product. (b) Similar assay steps achieved in a train of droplets containing 4 droplets (shown in the dashed box). The first droplet of the train contains the sample, magnetic beads and cortisol-HRP. The second and third droplets are washing droplets containing only PBS. The fourth droplet contains the TMB indicator. As the droplet train passes the magnetic trap (from left to right), the beads are captured and washed and finally re-dispersed in the final TMB indicator droplet where HRP catalyses colour changes.

supernatant from each reaction well was removed by manual pipetting, and the pellet of magnetic beads in each well was subsequently re-suspended by repeated gentle manual pipetting with each cortisol calibrant and cortisol-HRP. The plate was placed in a plate shaker for 15 minutes at $37{ }^{\circ} \mathrm{C}$ with shaking at $1200 \mathrm{rpm}$. Following this incubation step, the beads were recovered and then subjected to a wash step with a phosphate buffer. The beads were re-suspended with a TMB substrate (ThermoScientific, 1-Step Ultra TMB ELISA) followed by the addition of $2 \mathrm{M}$ sulphuric acid after 30 seconds. The reaction vessel was then placed on the magnetic plate stand, the resultant supernatant from each well was transferred to individual wells of a fresh clear flat-bottomed plate, and the absorbance was measured at $450 \mathrm{~nm}$.

\section{Portable droplet microfluidic platform for miniaturising ELISA}

Fig. 1b shows the heterogeneous assay steps carried out in a train of droplets. The droplet train contains four droplets for each measurement, i.e. a sample droplet (also containing the antibody coated beads and the competitor, cortisol-HRP), two washing droplets, and an indicator droplet containing the colorimetric indicator. One key design consideration of the droplet platform is the automation of the whole process, from sample collection, droplet generation, washing steps to optical detection and data logging.
We designed and fabricated a droplet microfluidic system to complete these procedures as illustrated in Fig. 2a. A 3Dprinted 12-line peristaltic micropump (Fig. 2b) was designed to drive all of the liquids and to produce the droplet trains. The sample liquid was aspirated via a cannula which can be inserted into a liquid cuvette or other sampling sites (e.g. saliva or blood vessel). The other liquids (magnetic bead suspension, cortisol-HRP solution, fluorocarbon oil (FC-40), TMB solution and PBS buffer) were also aspirated by the same pump from their separate storage sachets into a microfluidic chip. Sedimentation of magnetic particles from suspensions is a prevalent challenge in microfluidic systems. ${ }^{34}$ Here glycerol was added to the magnetic bead solution as an inert thickening agent (50:50 glycerol : PBS) which can decrease the rate of sedimentation without interfering with the bead chemistry.

The pump utilises a pulsed droplet generation technique presented earlier by Nightingale et al. ${ }^{12}$ but altered for the generation of four-droplet trains. The pulsed droplet generation technique has the advantage of robust generation of droplets with the designed droplet volume, contents and spacing invariant from changes in flow rates, viscosity and interfacial tensions, as demonstrated in Fig. S1. $\dagger$

In this device the grooves on the rotorhead of the pump and pump-line structures shown in the inset of Fig. $2 \mathrm{~b}$ deliver fluids in eight separate phases within one turn of the rotor- 
a)

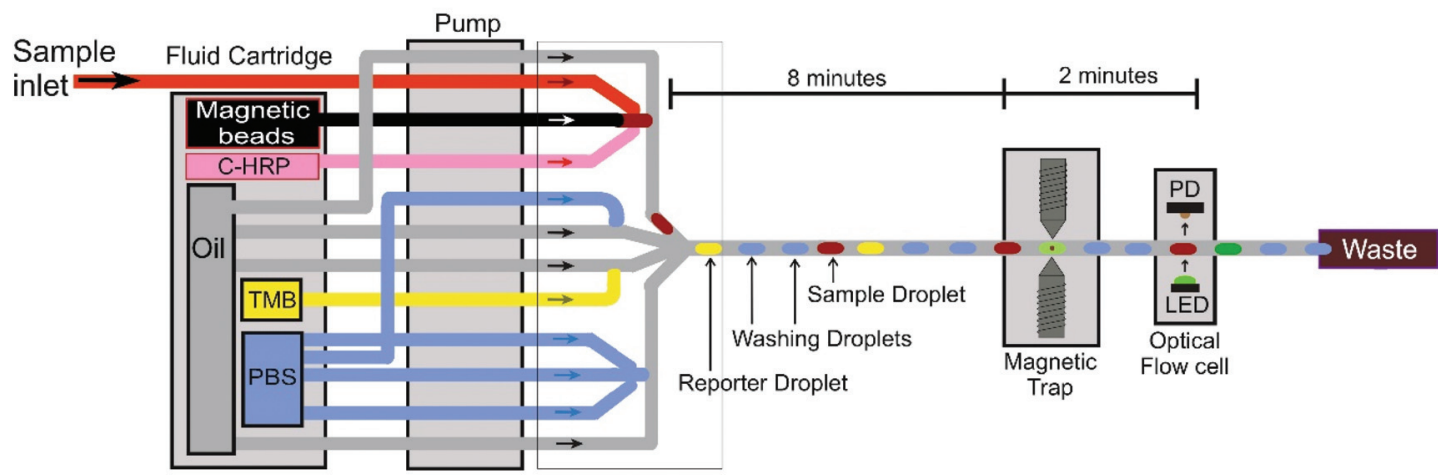

b)

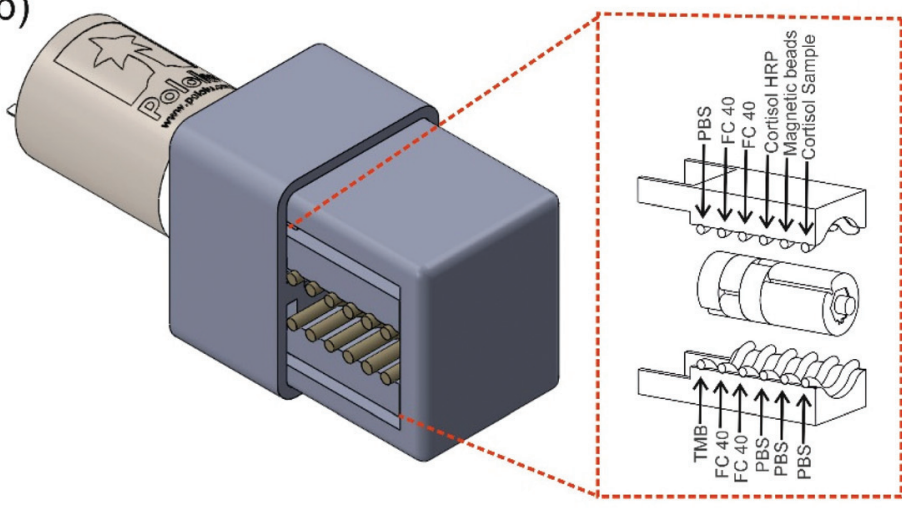

c)

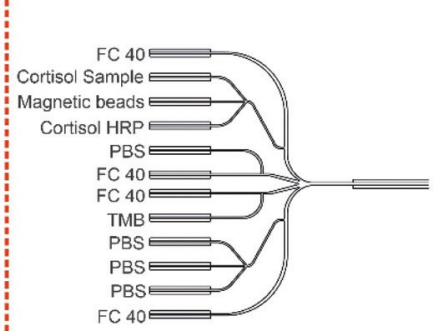

Fig. 2 Schematics of (a) the fluidic conduits and (b) the pump used to generate the four-droplet trains. (c) The design of the droplet generation chip. In (a), the magnetic bead suspension, C-HRP solution, FC-40 oil, TMB solution and PBS are stored in separate sachets in the fluid cartridge. PD: photodetector and amplifier.

head of the pump. The 1st phase is co-flow of the sample, bead suspension and cortisol-HRP, 2nd FC-40 oil, 3rd PBS, 4th FC-40 oil, 5th PBS, 6th FC-40 oil, 7th TMB, and 8th FC-40 oil. After the 2nd phase, the three aqueous inputs of the sample, magnetic beads and cortisol-HRP (represented by red food dye) are segmented into a droplet (called sample droplet) by the oil at a T-junction and finally moved into the main output channel of the chip. Then at the 4th and 6th phases, two PBS washing droplets are produced and enter the main outlet channel. Phase 8 produces the indicator droplet which is the last in a droplet train.

This whole process of droplet train production can be autonomously repeated with the rotation of the motor shaft of the pump. The droplet volume and oil gap can be 'hardcoded' from the design of the rotorhead and selection of inner diameters of the pump tubing.

The droplets exit the microfluidic chip into a polytetrafluoroethylene (PTFE) tubing maintaining the sequence of the train and flow for 8 minutes to allow the completion of anti-cortisol binding with cortisol/cortisol-HRP. These droplets then pass into the magnetic trap as shown in Fig. 2a. The magnetic tweezers extract the magnetic beads from the sample droplet, hold them in place to be washed by the following two buffer droplets, and finally re-disperse them into the reporter droplet. After two minutes of further reaction time, the reporter droplet is detected using an absorbance flow cell, ${ }^{41}$ with an absorbance inversely proportional to the cortisol concentration in the sample liquid. Finally, all of the droplets and oil flow into a waste sachet.

The microfluidic pump was designed and modelled using CAD software (SolidWorks), and 3D-printed in the VeroClear material using an Objet500 Connex3 polyjet printer (Stanford Marsh Ltd). A DC motor (499:1 Metal Gearmotor, 6V, RobotShop, UK) was attached to the chassis via M3 plastic screws and used to drive the rotorhead.

\section{Peristaltic micropump and droplet train generation}

The rotorhead of the pump was designed to deliver the four droplets in a train with approximate volumes: $1060 \mathrm{~nL}$ for droplets 1 and 3, and $550 \mathrm{~nL}$ for droplets 2 and 4, as the rotorhead pumps the tubing symmetrically on the top and bottom pump beds (Fig. 2b). The device was firstly tested for reproducible droplet generation at a low flow rate and low generation frequency (in this case $<1 \mathrm{~Hz}$ for each junction). Our pulsed droplet generation method can pump fixed volumes of aqueous and oil phases alternatively into each junction; therefore reproducible droplets can be generated at various flow rates and low generation frequency. ${ }^{12}$ Droplets generated were recorded both directly in the chip and off-chip at the PTFE tubing. The videos were further analysed using the Droplet 
a)

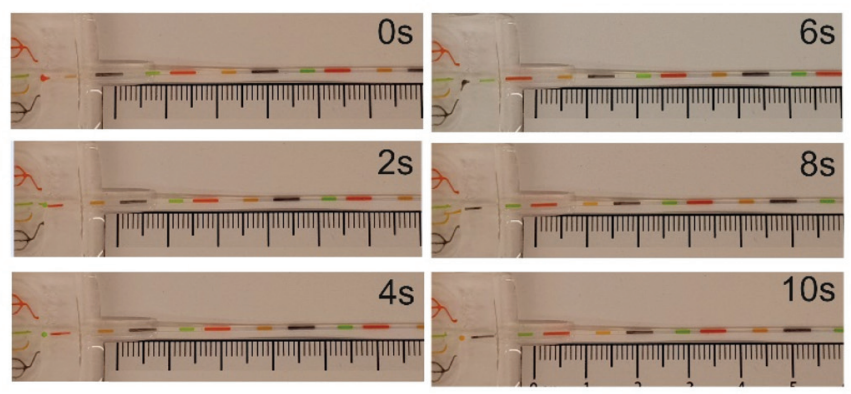

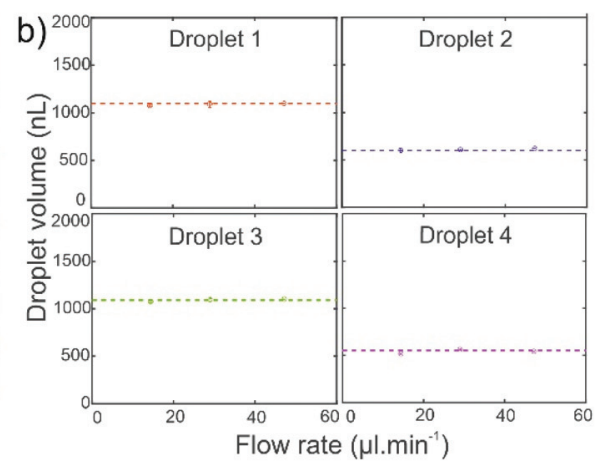

Fig. 3 Calibration of the droplet generation. (a) Step by step (0 to $10 \mathrm{~s}$ ) images of the droplet generation showing the microfluidic chip and exit PTFE tubing. (b) Droplet volume vs. flow rate of the droplet sequence (later used to carry out cortisol assay in droplets), demonstrating the droplet volume invariance to changes in the mean volumetric flow rate of the pulsed droplet generation regime.

Morphology and Velocimetry (DMV) software ${ }^{42}$ to characterise the droplet volume, generation rate and flow rate (up to $60 \mu \mathrm{L}$ $\mathrm{min}^{-1}$ ) as shown in Fig. 3. Fig. 3a shows the snapshots from a whole droplet train generation taking about $10 \mathrm{~s}$ in total. Further analysis of over 100 droplet trains at each tested flow rate is shown in Fig. 3b. The average volumes are $1060 \mathrm{~nL}$ for droplets 1 and 3 and $550 \mathrm{~nL}$ for droplets 2 and 4 . Coefficients of variation between droplet trains are consistently less than $2 \%$ (as indicated by the error bars in Fig. $3 \mathrm{~b}$ ) which compares favourably with previously reported droplet generation methods. ${ }^{43-45}$ This low coefficient of variation in the droplet volume was repeatable across many experiments (including varied viscosity of the fluids and different surface tensions by changing the concentration of the surfactant, as shown in the ESI in Fig. S1†) indicating the robust nature of the sampling and droplet generation method and ensuring synchronisation during the assay with our automatic magnetic tweezers. This method of pumping and droplet generation requires only a DC motor to drive all of the fluidics which leads to a small footprint of the device.

\section{Magnetic trap}

Once droplets are generated and the sample cortisol and cortisol-HRP complete the competitive binding process, an automated magnetic trap, shown in Fig. 4 (fabrication detailed in the ESI + ), is used to collect and shift the magnetic beads between droplets. As previously described by Pamme et al. ${ }^{46}$ extracting magnetic beads from a droplet requires a magnetic field strong and focused enough to overcome hydrodynamic and interfacial forces. Here the magnetic trap employs a pair of custom-made electromagnetic tweezers. The ferrite cores of the tweezers are located in two micro-machined groves and pinpoint to the PTFE tubing to focus the magnetic field. The choice of magnetic beads and the composition of the washing solution are also important which can affect the purification efficiency of the magnetic beads. Superparamagnetic particles (Dynabeads MyOne) are used because they do not retain "magnetic memory" ${ }^{46}$ in comparison to ferromagnetic particles which are permanently magnetized making resuspension during washing steps of the assay impossible. ${ }^{19}$ A detailed calibration was carried out on the magnetic field strength required to fully collect the magnetic particles, as shown in Fig. S2 in the ESI. $\dagger$ The capturing of magnetic beads at different currents, flowrates and bead quantities has also been quantified in Fig. S3 of the ESI. $\dagger$ An electric current of $0.7 \mathrm{~A}$ with a magnetomotive force of 140 ampere-turn, applied to a ferrite magnetic core of $3 \mathrm{~mm}$ diameter and $25 \mathrm{~mm}$ length, resulted in an approximate field strength of $11.2 \mathrm{mT}$ at the focusing area. This field strength was tested to be strong enough to collect the beads in the droplet or to break up the pellet from the droplet at a flow rate of $\leq 40 \mu \mathrm{L} \mathrm{min}^{-1}$ as seen under a microscope.

Accurate timing to trigger the magnetic trap is critical for the capture and re-dispersion of the magnetic beads. Here a pair of 'light gates' (Fig. 4a) and its control circuit were used to determine when to activate the electromagnetic tweezers to extract beads and when to deactivate to re-disperse the beads into the next droplet. These light gates are based on the previously described miniaturised in-line spectrophotometer. ${ }^{41}$ When a droplet passes through the PTFE tubing between the LED and photodetector, the light intensity measured by the optical detector shows a step change, which can trigger the on/ off of the magnetic field via an electronic circuit. Fig. $4 \mathrm{~b}$ shows a photograph of the automated magnetic trap mounted atop its control board, and Fig. 4c illustrates the system's operation, with raw data from a one-minute period of automated function (shorter period shown in Fig. 4d). The blue and green plots show the transmitted light reaching the inlet and outlet photodetectors, respectively (PD1 \& PD2). The electric current in the coils is represented by the red plot situated at the bottom of both Fig. 4c and d. As shown in Fig. 4c, to assist in bead capture the tweezers were activated immediately after its detection by the inlet detector and before the droplet arrived in the trap. The beads were then captured at the focusing point and two wash droplets were allowed to pass through to wash the beads. When the reporter droplet reached the trap, the beads were released for re-dispersion. As the beads must overcome the surface tension of the droplet interface to re-disperse 
a)

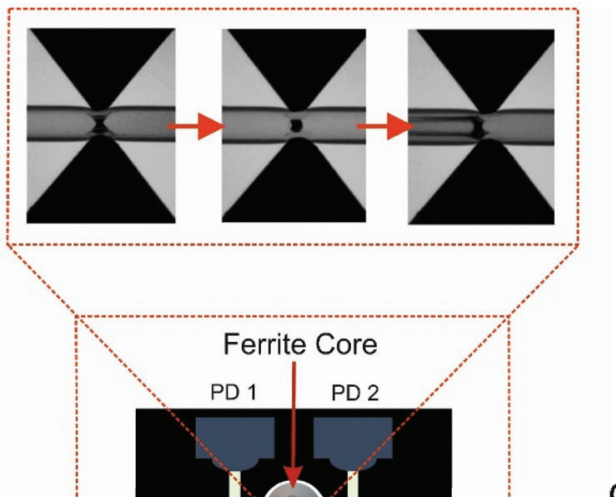

Fig. 4 Autonomous magnetic bead trap. (a) Schematics of the magnetic bead trap with a flow cell featuring two 'light gates' (spectrophotometers) which are used to determine when to activate the trap to capture or deactivate to re-disperse the beads into a new droplet. The top inset shows the capture, retention and re-distribution of the magnetic beads. (b) Photograph of the fabricated magnetic bead-trap. (c) Light intensities detected by photodetectors 1 and 2 (PD1 and PD2). The red line shows the actuation (high level) and de-actuation (low level) of the magnetic field. (d) Close up of the data trace in (c). Detector 1 (blue trace) determines when to activate the trap and photodetector 2 (green trace) determines when to deactivate the trap, re-dispersing the bead pellet in a droplet. (e) Washing efficiency of the magnetic beads. Here highly concentrated Rose Bengal dye was used to indicate the colour change of the droplet after washing. FC40 oil with $0.1 \%$ PFPE-PEG surfactant was used as the carrier phase (to aid the breakup of bead pellets from the first droplet).

safely, releasing of the beads was only triggered when the outlet detector senses the approaching of the reporter droplet, while it still fills the centre of the trap.

\section{Washing efficiency}

In a competitive ELISA, removal of the unbound analyte is necessary as otherwise they will interfere with the following measurement. This step is equally necessary for a droplet flow based assay. There are different approaches to achieving these washing steps. One approach is to collect the magnetic beads with the magnetic tweezers into the next washing droplets and re-disperse them, and then re-collect and send them to another washing or indicator droplet. However for a flowing train of droplets, this will require multiple pairs of tweezers to c)

d)

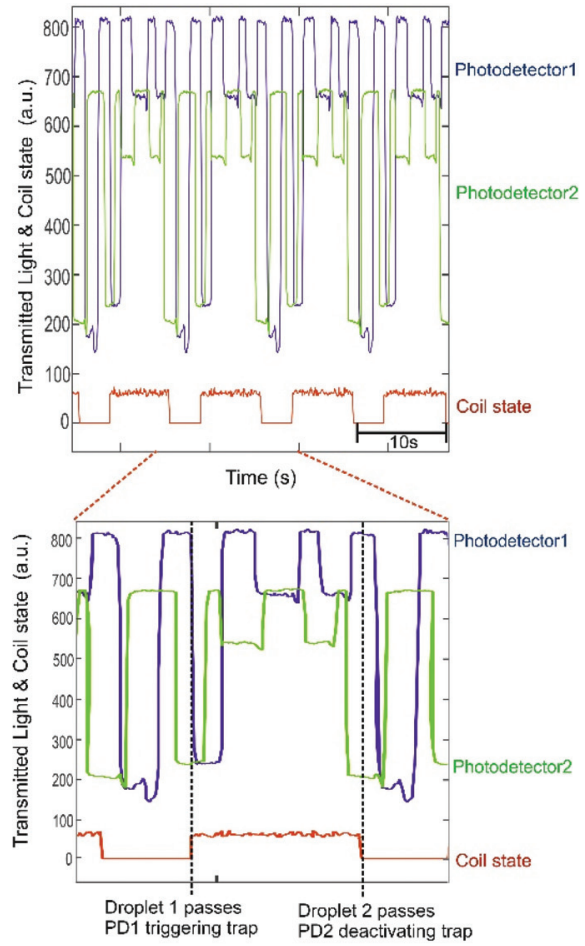

e)

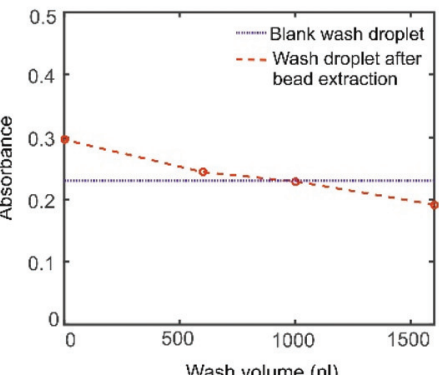


remained energised and the bead 'pellet' was held stationary while washing droplets passed through the trap. As the washing droplets passed through the bead pellet, the bead pellet was observed to deform indicating partial re-dispersion. Four varieties of droplet trains with varying washing volumes were tested (Fig. S5 in the ESI $\dagger$ ) with the absorbance of the redispersed purified beads shown in Fig. 4e (red line) as compared to the pure washing droplet (blue line). In train 1 the beads were simply captured from the first droplet and re-dispersed ( $0 \mathrm{~nL}$ wash volume) into the indicator droplet. For trains 2 and 3 the beads were washed in a single droplet of 550 $\mathrm{nL}$ and $1060 \mathrm{~nL}$, respectively. In train 4, two wash droplets totalling $1610 \mathrm{~nL}$ volume were used. This experiment indicated that purification regimes 3 and 4 provide thorough washing with negligible remaining dye in the indicator droplet. Calculations from the absorbance change from regime 4 in Fig. 4e indicate that the loss of beads is about $50 \mathrm{ng}$ per train after two washing steps, equivalent to $16 \%$ of the total weight of magnetic beads. The loss of beads will reduce the strength of the signal in the final indicator droplet. However as the proportion of bead loss was consistent for all of the droplet trains once the washing scheme was fixed, the accuracy of final results was not affected as discussed later in Fig. 6.

\section{Inline spectrophotometer for absorption detection}

An inline spectrophotometer, shown schematically in Fig. 5a, ${ }^{11,41,47}$ was used to characterise the colour change in the indicator droplet, where TMB was catalysed by the cortisolHRP from yellow-green to blue-green. A $456 \mathrm{~nm}$ blue LED (Cree PLCC4 1-in-1 SMD LED CLM4B-BKW/GKW) and a photodetector (PD, TSL257. Farnell, UK) pair was installed in a micro-milled PMMA holder forming the spectrophotometer to quantify the absorbance. The concentration can then be calcu- lated from a pre-determined calibration curve. Fig. $5 \mathrm{~b}$ and $\mathrm{c}$ show the intensity of light from the inline spectrophotometer as a function of time for cortisol standards from $3.175 \mathrm{ng}$ $\mathrm{mL}^{-1}$ and $100 \mathrm{ng} \mathrm{mL}^{-1}$ respectively. The reporter droplet containing $100 \mathrm{ng} \mathrm{mL} \mathrm{m}^{-1}$ cortisol (Fig. 5c) showed less colour development than $3.175 \mathrm{ng} \mathrm{mL}^{-1}$ ones (Fig. 5b) as should be for a competitive heterogeneous assay. It is to be noted that the droplets were carried by oil which can prevent direct contact from the magnetic beads or molecules in the droplet to the optical surface in the spectrophotometer; therefore the spectrophotometer can be used continuously without cleaning and there is no cross-contamination between samples.

\section{Comparison of bulk assay and droplet microfluidics}

The assay was first carried out in triplicate in a 96-well plate procedure ('bulk assay') with standard calibrants consisting of cortisol from $3.175 \mathrm{ng} \mathrm{mL^{-1 }}$ to $100 \mathrm{ng} \mathrm{mL}^{-1}$ in PBS buffer. Fig. 6a shows the final assay results as a calibration curve of absorbance versus cortisol concentration. The basal serum free cortisol level in healthy individuals is typically $4.7125 \mathrm{ng} \mathrm{mL}$ $\pm 0.725 \mathrm{ng} \mathrm{mL}{ }^{-1}$ as estimated by Coolens' method. ${ }^{48}$ The assay carried out by the 96-well plate procedure was designed to display a good linear range between $3.175 \mathrm{ng} \mathrm{mL}^{-1}$ and $50 \mathrm{ng}$ $\mathrm{mL}^{-1}$ with a distinct increase in variability at $100 \mathrm{ng} \mathrm{ml}^{-1}$. As expected from a competitive assay, the measured absorbance in Fig. 6a is inversely proportional to the concentration of cortisol in the sample. The colour intensity began to reach the saturation state for sample cortisol concentrations lower than $3.175 \mathrm{ng} \mathrm{mL}^{-1}$. However, the relative standard deviation (RSD) of the assay is particularly large for some of the points (as high as $80 \%$ ) even after repeated assays. This may be due to human factors such as varied mixing in the well plate. Also note this procedure took a considerable amount of time to
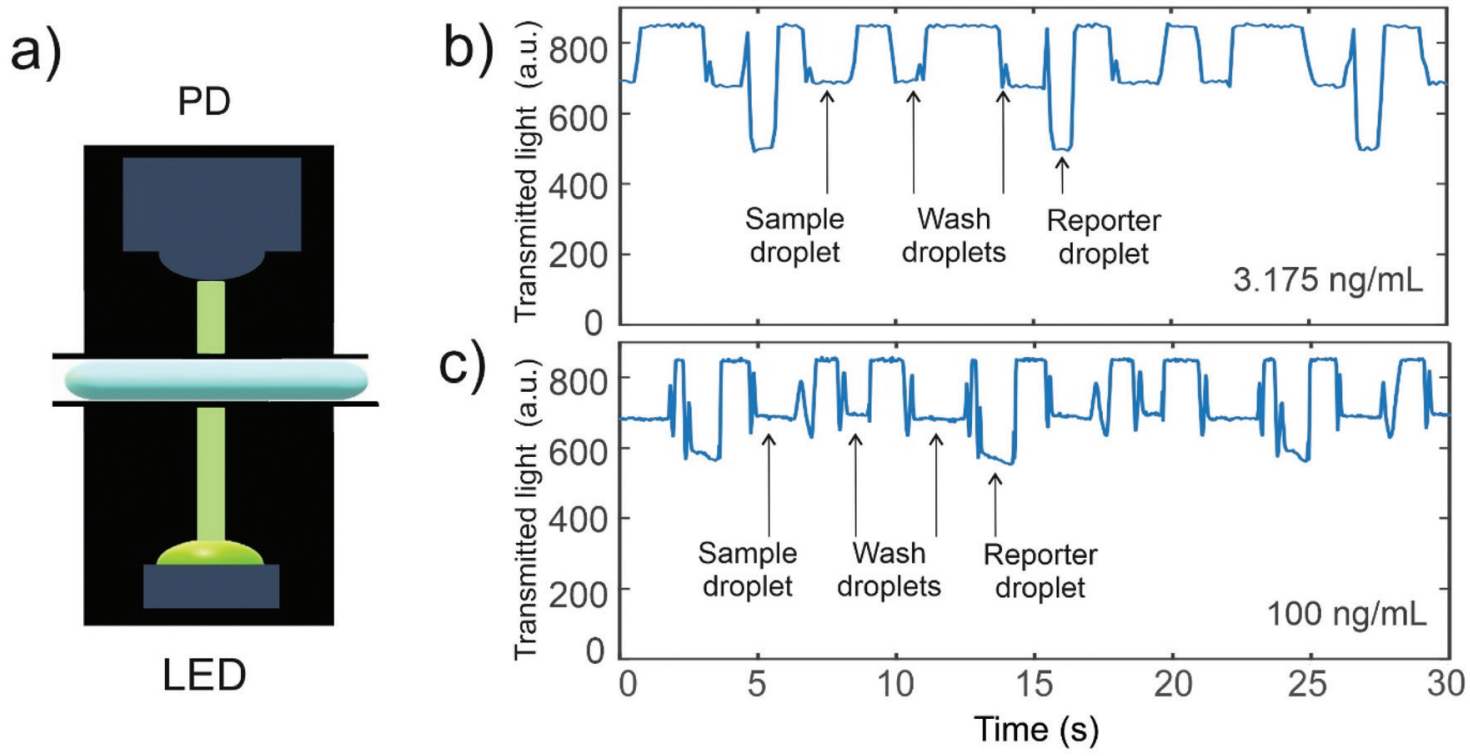

Fig. 5 Inline spectrophotometer for absorption detection. (a) Schematics of the flow cell. (b) and (c) Light intensity readout of the droplet trains containing cortisol standards $3.175 \mathrm{ng} \mathrm{mL}^{-1}$ in (b) and $100 \mathrm{ng} \mathrm{mL}^{-1}$ in (c). 
a)

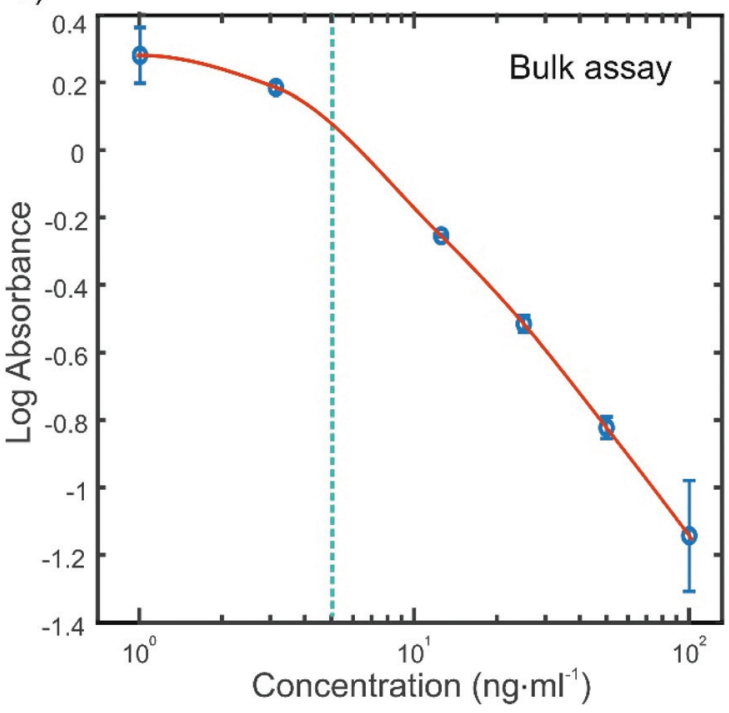

b)

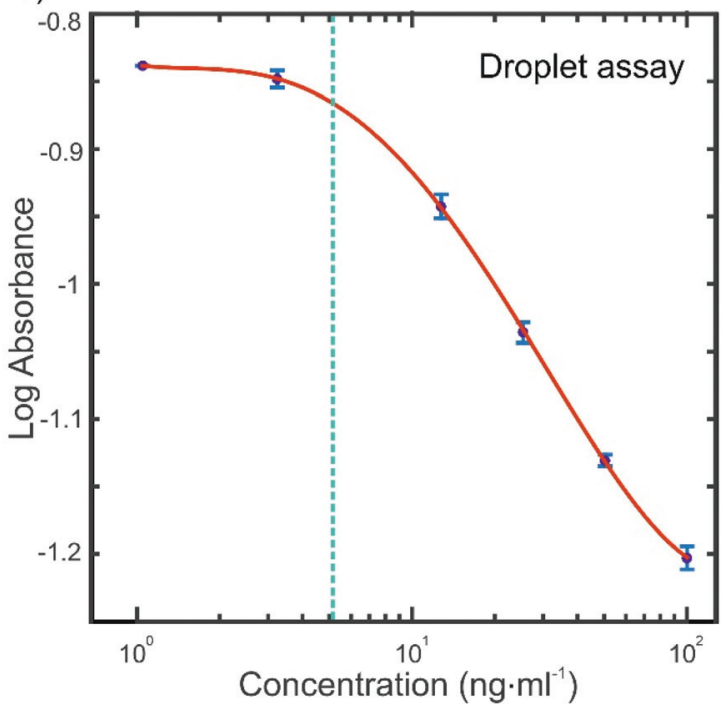

Fig. 6 Calibration curves for the competitive cortisol assay. (a) Results from the bulk laboratory procedure (FLUOstar Omega; BMG LABTECH) and (b) results from the droplet microfluidic platform constructed from a PBS buffer. The noted dotted line shows that basal serum free cortisol levels in healthy individuals are typically $4.7125 \mathrm{ng} \mathrm{ml}^{-1} \pm 0.725 \mathrm{ng} \mathrm{ml}^{-1}$ as estimated by Coolens' method. ${ }^{48}$

complete ( $\sim 4$ hours for each assay) due to multiple washing and mixing steps as well as lengthy incubation times.

After bulk testing, the assay was transferred to the droplet platform. Serial dilutions of cortisol were generated from 100 ng $\mathrm{mL}^{-1}$ to $3.175 \mathrm{ng} \mathrm{mL}^{-1}$ in a PBS buffer. The cortisol-HRP was kept constant at $100 \mathrm{ng} \mathrm{mL}^{-1}$. During the test the pump was controlled to aspirate the sample, reagents and oil as described in the Assays and methods section. The device produces one droplet train every $10 \mathrm{~s}$ (each train corresponding to one measurement point).

The droplet trains flow in the tubing at room temperature for a first incubation time of $8 \mathrm{~min}$, which was found sufficient for the competitive binding process to complete. This incubation time is much shorter than required in a bulk assay of around $30 \mathrm{~min}$. We attribute it to the continued and thorough mixing by vortex advection in droplets. The beads were then extracted by the magnetic tweezers, washed with buffer droplets and re-dispersed in the final TMB droplet with the cortisol-HRP catalysing the colour change for $2 \mathrm{~min}$ (in the flowing droplets). This colour change was then measured using an inline spectrophotometer with measurement from example droplet trains shown in Fig. 5b and c and collectively shown in Fig. $6 \mathrm{~b}$ with each concentration containing 10 repeats of droplet trains.

The absorbance value in Fig. $6 \mathrm{~b}$ is systematically lower than the reading from the well plate in Fig. 6a, due to the very different light path lengths used in the two detectors $(0.5 \mathrm{~mm}$ in droplet device versus $5 \mathrm{~mm}$ in a well plate reader). However both the results in Fig. $6 \mathrm{a}$ and b show a qualitatively similar trend over the concentration ranges measured. In Fig. 6b, the RSD is much lower (less than 1\%) than that of the well plate based assay, due to the automated process, accurate volu- metric control of the fluids and enhanced mixing. Additional samples (containing $12.5 \mathrm{ng} \mathrm{ml}^{-1}$ and $100 \mathrm{ng} \mathrm{ml}^{-1}$ cortisol) were tested and given the recovered value from the standard curve of $14.96 \mathrm{ng} \mathrm{ml}^{-1}$ with a standard deviation of $1.33 \%$ and $102.8 \mathrm{ng} \mathrm{ml}^{-1}$ with a standard deviation of $1.05 \%$, meaning that the error is $19.7 \%$ for the former and less than $2.8 \%$ for the latter. The total volume of all fluids required for each assay carried out in the droplet platform is $\sim 3 \mu \mathrm{L}$ for each measurement. As a comparison, a lab-based assay requires a working volume of $150 \mu \mathrm{L}$ each for sample and reagent, and equal or more volumes of washing buffer.

\section{Discussion and conclusions}

We designed, fabricated, and tested the prototype of a droplet microfluidic device capable of performing an autonomous and continuous ELISA in droplets. A multi-line peristaltic pump enabled sample collection and continuous generation of droplet trains without the need for complicated valves or a robotic pipetting system. This device can collect samples directly from a reservoir or potentially from tissue/bloodstream via a microdialysis probe, requiring a small sample volume for each analysis (350 $\mathrm{nl})$, and providing a near real time assay and results. We have demonstrated that the device can perform high frequency measurement for cortisol and has the potential to run assays continuously, with a detection range comparable to those of well plate-based assays and with smaller errors by obviating human operation.

The droplet platform is capable of forming a droplet train approximately every 10 seconds. The sample-to-signal time is about $10 \mathrm{~min}$ at room temperature determined by the competi- 
tive binding process, which is significantly lower than those of well plate based assays where one measurement takes about $4 \mathrm{~h}$ and requires intensive manual handling. The low volume of reagent consumption is particularly important for multiple time measurements required for long-term monitoring. Overall, the device has a small footprint and consumes low power, and contains modular and adaptable components, making it a platform technology to perform complex assays and for monitoring at the point-of-care.

\section{Conflicts of interest}

The authors declare the following competing financial interests: Xize Niu is the founder of SouthWestSensor Ltd, a company commercializing droplet-based sensor technologies. Brett Warren, Kyriacos Makris, Sharon Coleman and Sammerul Hassan are all employees of the same company. No potential conflicts of interest exist for the other co-authors.

\section{Acknowledgements}

We thank the Engineering and Physical Sciences Research Council, UK (EP/M012425/1), NIHR BRC Global NAMRIP, and Innovate UK (103736) for funding this work.

\section{Notes and references}

1 M. L. Rogers and M. G. Boutelle, Real-time clinical monitoring of biomolecules, Annu. Rev. Anal. Chem., 2013, 6, 427-453.

2 C. H. Ahn, et al., Disposable smart lab on a chip for point-of-care clinical diagnostics, Proc. IEEE, 2004, 92(1), 154-173.

3 S. A. Soper, et al., Point-of-care biosensor systems for cancer diagnostics/prognostics, Biosens. Bioelectron., 2006, 21(10), 1932-1942.

4 J. Wang, Electrochemical biosensors: Towards point-of-care cancer diagnostics, Biosens. Bioelectron., 2006, 21(10), 1887-1892.

5 G. S. Wilson and R. Gifford, Biosensors for real-time in vivo measurements, Biosens. Bioelectron., 2005, 20(12), 23882403.

6 J. M. Morais, F. Papadimitrakopoulos and D. J. Burgess, Biomaterials/tissue interactions: possible solutions to overcome foreign body response, AAPS J., 2010, 12(2), 188-196.

7 N. Wisniewski, F. Moussy and W. M. Reichert, Characterization of implantable biosensor membrane biofouling, Fresenius' J. Anal. Chem., 2000, 366(6), 611-621.

8 N. Wisniewski and M. Reichert, Methods for reducing biosensor membrane biofouling, Colloids Surf., B, 2000, 18(3), 197-219.

9 C. M. B. Ho, et al., 3D printed microfluidics for biological applications, Lab Chip, 2015, 15(18), 3627-3637.
10 A. A. Yazdi, et al., 3D printing: an emerging tool for novel microfluidics and lab-on-a-chip applications, Microfluid. Nanofluid., 2016, 20(3), 50.

11 S.-U. Hassan, A. M. Nightingale and X. Niu, Micromachined optical flow cell for sensitive measurement of droplets in tubing, Biomed. Microdevices, 2018, 20(4), 92-92.

12 A. M. Nightingale, et al., Phased peristaltic micropumping for continuous sampling and hardcoded droplet generation, Lab Chip, 2017, 17(6), 1149-1157.

13 M. Abdelgawad and A. R. Wheeler, The Digital Revolution: A New Paradigm for Microfluidics, Adv. Mater., 2009, 21(8), 920-925.

14 A. Koh, et al., A soft, wearable microfluidic device for the capture, storage, and colorimetric sensing of sweat, Sci. Transl. Med., 2016, 8(366), 366ra165-366ra165.

15 J. M. Anderson, Biological Responses to Materials, Annu. Rev. Mater. Res., 2001, 31(1), 81-110.

16 A. Campbell and C. Wu, Chronically Implanted Intracranial Electrodes: Tissue Reaction and Electrical Changes, Micromachines, 2018, 9(9), 430.

17 A. M. Nightingale, et al., Monitoring biomolecule concentrations in tissue using a wearable droplet microfluidicbased sensor, Nat. Commun., 2019, 10(1), 2741.

18 W. Liu, et al., SlipChip for immunoassays in nanoliter volumes, Anal. Chem., 2010, 82(8), 3276-3282.

19 A. Ali-Cherif, et al., Programmable Magnetic Tweezers and Droplet Microfluidic Device for High-Throughput Nanoliter Multi-Step Assays, Angew. Chem., Int. Ed., 2012, 51(43), 10765-10769.

20 D. Ferraro, et al., Microfluidic platform combining droplets and magnetic tweezers: application to HER2 expression in cancer diagnosis, Sci. Rep., 2016, 6, 25540.

21 B. Teste, et al., A low cost and high throughput magnetic bead-based immuno-agglutination assay in confined droplets, Lab Chip, 2013, 13(12), 2344-2349.

22 A. Kaushik, et al., Recent advances in cortisol sensing technologies for point-of-care application, Biosens. Bioelectron., 2014, 53, 499-512.

23 E. R. De Kloet, M. Joëls and F. Holsboer, Stress and the brain: from adaptation to disease, Nat. Rev. Neurosci., 2005, 6(6), 463-475.

24 R. Gatti, et al., Cortisol assays and diagnostic laboratory procedures in human biological fluids, Clin. Biochem., 2009, 42(12), 1205-1217.

25 A. Levine, et al., Measuring cortisol in human psychobiological studies, Physiol. Behav., 2007, 90(1), 43-53.

26 K. Gracie, et al., Detection of cortisol in serum using quantitative resonance Raman spectroscopy, Anal. Methods, 2017, 9(10), 1589-1594.

27 D. Corbalán-Tutau, et al., Daily profile in two circadian markers "melatonin and cortisol" and associations with metabolic syndrome components, Physiol. Behav., 2014, 123, 231-235.

28 F. Holsboer and M. Ising, Stress Hormone Regulation: Biological Role and Translation into Therapy, Annu. Rev. Psychol., 2010, 61(1), 81-109. 
29 D. Y. Lee, E. Kim and M. H. Choi, Technical and clinical aspects of cortisol as a biochemical marker of chronic stress, BMB Rep., 2015, 48(4), 209-216.

30 B. S. McEwen, Physiology and Neurobiology of Stress and Adaptation: Central Role of the Brain, Physiol. Rev., 2007, 87(3), 873-904.

31 B. S. McEwen, Cortisol, Cushing's Syndrome, and a Shrinking Brain-New Evidence for Reversibility, J. Clin. Endocrinol. Metab., 2002, 87(5), 1947-1948.

32 O. Edwards, et al., Changes In cortisol metabolism following rifampicin therapy, Lancet, 1974, 304(7880), 549551.

33 J. L. Barron, et al., Hypothalamic Dysfunction in Overtrained Athletes*, J. Clin. Endocrinol. Metab., 1985, 60(4), 803-806.

34 C. A. Czeisler, et al., Episodic 24-Hour Cortisol Secretory Patterns in Patients Awaiting Elective Cardiac Surgery, J. Clin. Endocrinol. Metab., 1976, 42(2), 273-283.

35 D. Giavarina and G. Lippi, Blood venous sample collection: Recommendations overview and a checklist to improve quality, Clin. Biochem., 2017, 50(10), 568-573.

36 P. R. Brezina, E. Haberl and E. Wallach, At home testing: optimizing management for the infertility physician, Fertil. Steril., 2011, 95(6), 1867-1878.

37 O. Wallin, et al., Preanalytical venous blood sampling practices demand improvement-A survey of test-request management, test-tube labelling and information search procedures, Clin. Chim. Acta, 2008, 391(1), 91-97.

38 M. Plebani and G. Lippi, Is laboratory medicine a dying profession? Blessed are those who have not seen and yet have believed, Clin. Biochem., 2010, 43(12), 939-941.
39 M. Plebani, et al., Quality indicators to detect pre-analytical errors in laboratory testing, Clin. Chim. Acta, 2014, 432, 4448.

$40 \mathrm{~V}$. Bhat, et al., Analysis of laboratory sample rejections in the pre-analytical stage at an oncology center, Clin. Chim. Acta, 2012, 413(15), 1203-1206.

41 S. Hassan, A. M. Nightingale and X. Niu, Continuous measurement of enzymatic kinetics in droplet flow for point-of-care monitoring, Analyst, 2016, 141(11), 3266-3273.

42 A. S. Basu, Droplet morphometry and velocimetry (DMV): a video processing software for time-resolved, label-free tracking of droplet parameters, Lab Chip, 2013, 13(10), 1892-1901.

43 S. H. Tan, et al., Thermally controlled droplet formation in flow focusing geometry: formation regimes and effect of nanoparticle suspension, J. Phys. D: Appl. Phys., 2008, 41(16), 165501.

44 S. Y. Jung, S. T. Retterer and C. P. Collier, On-demand generation of monodisperse femtolitre droplets by shapeinduced shear, Lab Chip, 2010, 10(20), 2688-2694.

45 A. R. Abate, et al., Synthesis of Monodisperse Microparticles from Non-Newtonian Polymer Solutions with Microfluidic Devices, Adv. Mater., 2011, 23(15), 1757-1760.

46 N. Pamme, Magnetism and microfluidics, Lab Chip, 2006, 6(1), 24-38.

47 S.-u. Hassan, A. M. Nightingale and X. Niu, Optical Flow Cell for Measuring Size, Velocity and Composition of Flowing Droplets, Micromachines, 2017, 8(2), 58.

48 R. I. Dorin, et al., Validation of a simple method of estimating plasma free cortisol: Role of cortisol binding to albumin, Clin. Biochem., 2009, 42(1), 64-71. 